\title{
Quasi-elastic backscattering of ${ }^{6,7} \mathrm{Li}$ on light, medium and heavy targets at near- and sub-barrier energies
}

\author{
K. Zerva ${ }^{1}$, A. Pakou ${ }^{1, a}$, N. Patronis ${ }^{1}$, P. Figuera ${ }^{2}$, A. Musumarra ${ }^{2}$, A. Di Pietro ${ }^{2}$, M. Fisichella ${ }^{2}$, T. Glodariu ${ }^{3}$, \\ M. La Commara ${ }^{4}$, M. Lattuada ${ }^{2}$, M. Mazzocco ${ }^{5}$, M.G. Pellegriti ${ }^{2}$, D. Pierroutsakou ${ }^{6}$, A.M. Sanchez-Benitez ${ }^{7}$, \\ V. Scuderi², E. Strano ${ }^{2}$, and K. Rusek ${ }^{8,9}$ \\ 1 Department of Physics and HINP, The University of Ioannina, 45110 Ioannina, Greece \\ 2 INFN-LNS and University of Catania, Italy \\ 3 "Horia Hulubei" National Institute of Physics and Nuclear Engineering, Romania \\ 4 Dipartimento di Scienze Fisiche and INFN, Sezione di Napoli, via Cinthia, I-80126 Napoli, Italy \\ ${ }^{5}$ Dipartimento di Fisica, INFN, I35131 Padova, Italy \\ 6 INFN, Sezione di Napoli, via Cinthia, I-80126 Napoli, Italy \\ 7 Dapartamento di Fisica Atomica Molecular y Nuclear, Universidad de Huelva, E-21071 Huelva, Spain \\ 8 Heavy Ion Laboratory, University of Warsaw, Warsaw, Poland \\ 9 Department of Nuclear Reactions, The Andrzej Soltan Institute for Nuclear Studies, Warsaw, Poland
}

Received: 23 May 2012 / Revised: 6 July 2012

Published online: 27 July 2012

(C) The Author(s) 2012. This article is published with open access at Springerlink.com Communicated by N. Alamanos

\begin{abstract}
We have performed precision elastic backscattering measurements for the weakly bound nuclei ${ }^{6,7} \mathrm{Li}$ on the medium and heavy mass targets ${ }^{58} \mathrm{Ni},{ }^{116,120} \mathrm{Sn}$ and ${ }^{208} \mathrm{~Pb}$ at sub- and near-barrier energies $\left(E_{c . m .} / V_{\text {bar }}=0.6\right.$ to 1.3$)$. Excitation functions of elastic scattering cross-sections have been measured at $\pm 160^{\circ}$ and $\pm 170^{\circ}$ and the corresponding ratios to Rutherford scattering and relevant barrier distributions have been extracted. These measurements complement recent work on a ${ }^{28} \mathrm{Si}$ target, for probing systematically the potential at sub- and near-barrier energies and relevant reaction mechanisms, while indicate a possible breakdown of the dispersion relation.
\end{abstract}

\section{Introduction}

Over the last decade, considerable interest has been focused on the energy dependence of the optical potential at sub- and near-barrier energies for reactions involving weakly bound nuclei. In this case a new type of the optical potential threshold anomaly is observed [1-5]. The presence or a possible absence of the so-called "breakup threshold anomaly" is a question of paramount importance. It may challenge our current theoretical understanding of low-energy reaction dynamics involving weakly bound nuclei and modify our current understanding of the genesis and production rate of some nuclei produced in stellar explosions. Therefore our efforts should be intensified in order to determine the potential till very low energies, the main ingredient for predicting safely reaction cross-sections. However, two are the main inherent difficulties in such studies performed at low energies. The lack of sensitivity for obtaining the optical potential parameters and the strong couplings to transfer and breakup which al-

\footnotetext{
a e-mail: apakou@cc.uoi.gr
}

ter our view of the well-known potential for tightly bound nuclei. Variations on the ratio of transfer versus breakup from target to target for weakly bound projectiles may be reflected as variations on the energy trend of the optical potential. Such a case was already reported as a possibility by Lubian et al. [6] via polarization potential calculations. For further understanding this subject and being able to energy map the potential, other complementary means to the conventional techniques should be also adopted. As such, the application of elastic backscattering [7] was recently suggested in $[8,9]$, while its role for probing properties of the optical potential and the structure of the involved nuclei has been extensively demonstrated in recent years [10-17].

The elastic backscattering, as a tool for probing the energy dependence of the potential at near- and sub-barrier energies, was first applied to the weakly bound nuclei ${ }^{6} \mathrm{Li}$ and ${ }^{7} \mathrm{Li}$ scattered by the light target ${ }^{28} \mathrm{Si}[8,9]$. Previous inconsistencies $[18,19]$ with regard to the new potential anomaly, observed in this system, were clarified.

Our goal in this work is to systematically probe the potential and the importance of coupling effects at near- 
barrier energies by extending our backscattering measurements to medium and heavy targets, namely to ${ }^{58} \mathrm{Ni},{ }^{116,120} \mathrm{Sn}$ and ${ }^{208} \mathrm{~Pb}$. Other measurements of elastic and quasi-elastic backscattering barrier distributions for weakly bound nuclei have been reported previously for heavy targets: ${ }^{6,7} \mathrm{Li}+{ }^{208} \mathrm{~Pb}[20],{ }^{6} \mathrm{Li}+{ }^{144,146} \mathrm{Sm}[21,22]$ and ${ }^{6} \mathrm{Li}+{ }^{232} \mathrm{Th}[23]$ with the emphasis on the coupling effects rather than the energy dependence of the optical potential. The choice of almost spherical targets in the present case was based mainly on our intention to probe the potential without any strong interference from the structure of the involved nuclei.

The optical-model analysis for probing the optical potential as a function of energy was done by using for the real and imaginary part a BDM3Y1 interaction [24] but with different normalization factors, that is assuming the same radial dependence for the two parts of the optical potential. As it was shown in [18] at low energies and almost "spherical" targets, the assumption of the same radial dependence while it reduces the fitting parameters with obvious benefit, does not affect the quality of the fit. For the folding procedure, density distributions for the target nuclei were taken from electron scattering data in a conventional two- or three-parameter Fermi model [25]. A phenomenological model according to Bray et al. [26] and Hartree Fock calculations obtained by Trache et al. [27] were adopted for the densities of the projectiles ${ }^{6} \mathrm{Li}$ and ${ }^{7} \mathrm{Li}$, respectively. Finally the code ECIS [28] was used for reanalyzing elastic scattering differential cross-sections of previous measurements and to predict excitation functions of elastic scattering at backward angles. Continuum Discretized Coupled Channel (CDCC) calculations are presented for all the systems and the differential crosssections were obtained by using the code FRESCO [29] for probing couplings to continuum.

\section{Experimental details and data reduction}

Beams of ${ }^{6,7} \mathrm{Li}$ ions were delivered by the SMP Tandem accelerator of LNS in Catania at $0.5 \mathrm{MeV}$ energy steps in the energy range 0.6 to $1.3 V_{\text {bar }}$. Beam currents were of the order of 5 to $20 \mathrm{nA}$ depending on energy. The beams impinged on $\sim 200 \mu \mathrm{g} / \mathrm{cm}^{2}$ thick self-supporting ${ }^{58} \mathrm{Ni}$, ${ }^{116,120} \mathrm{Sn},{ }^{208} \mathrm{~Pb}$ targets, with the target frame fixed perpendicular to the beam direction. The overall normalization was estimated via a measurement of the Rutherford scattering in two silicon detectors set at \pm 20 degrees. Excitation functions of (quasi-)elastic backscattering events were recorded in four telescopes consisting of $10 \mu \mathrm{m}$ and $2000 \mu \mathrm{m}$ silicon detectors, set at \pm 160 and \pm 170 degrees. It should be clarified here that for ${ }^{6} \mathrm{Li}$ the excitation functions are determined via pure elastic scattering data while for ${ }^{7} \mathrm{Li}$ on $\mathrm{Ni}$ and $\mathrm{Sn}$ targets, quasi-elastic events were recorded consisting of elastic and inelastic scattering to the $1 / 2^{-}$first excited state of ${ }^{7} \mathrm{Li}$.

Ratios of the (quasi-)elastic cross-sections to Rutherford were formed as follows:

$$
\frac{\sigma_{e l}}{\sigma_{\text {Ruth }}}\left(160^{\circ}\right)=\frac{N_{160^{\circ}}}{N_{20^{\circ}}} \frac{\sigma_{\text {Ruth }}\left(20^{\circ}\right)}{\sigma_{R u t h}\left(160^{\circ}\right)} \frac{\Omega_{20^{\circ}}}{\Omega_{160^{\circ}}},
$$

where $N_{20^{\circ}}$ and $N_{160^{\circ}}$ are the (quasi-)elastic scattering total counts in the forward and backward detectors, and $\Omega_{20^{\circ}}$ and $\Omega_{160^{\circ}}$ are their respective solid angles. The ratio of the solid angles was determined as $0.0173 \pm 0.0001$ from the data at the lowest energies where the scattering is pure Rutherford for both forward and backward detectors. Therefore, the only major error involved in the above relation is the statistical error which at most of the energies was less than $2 \%$, except at the highest ones where it did not exceed the limit of $6 \%$. Ratios at $170^{\circ}$, were also deduced in the same way and finally mean ratios between 160 and 170 degrees were formed. The results are presented in fig. 1. The corresponding barrier distributions were determined by the following relation [7]:

$$
D_{\mathrm{el}}(E)=-\frac{\mathrm{d}}{\mathrm{d} E}\left[\sqrt{\frac{\mathrm{d} \sigma_{e l}}{\mathrm{~d} \sigma_{\text {Ruth }}}(E)}\right],
$$

using a point-difference formula for extracting the derivative. While this relation refers to elastic scattering it was also used for our quasi-elastic scattering data, since differences between elastic and quasi-elastic are unimportant, for easiness of comparisons between the barriers of the two projectiles. Our barrier distribution results are presented in figs. 2 and $4-8$. Results for ${ }^{116} \mathrm{Sn}$ were very similar like the ones for ${ }^{120} \mathrm{Sn}$ (fig. 9) and are not presented.

\section{Optical-model analysis}

The optical-model analysis is described in detail for the system ${ }^{6} \mathrm{Li}+{ }^{58} \mathrm{Ni}$, which is used as the main example for demonstrating the sensitivity and usefulness of the technique for probing the potential. As it was said before, the backscattering technique acts complementary to the traditional elastic scattering one, for probing accurately the energy dependence of the potential at near-barrier energies, extend it to sub-barrier energies and trace the type of the potential threshold anomaly. All the other systems are treated in the same way with emphasis on a final comparison between the potentials. The linear segment model for the imaginary part of the optical potential, $W(E)$, was applied according to G.R. Satchler [30]. This has the advantage of giving a simple analytic form for the dispersion relation, while it gives results close to more physical, smooth functions for $W(E)$. The starting point is the imaginary potential, which is described in the following by lines drawn from higher to lower energies. The real potential is calculated via dispersion relations. In the case of the ${ }^{7} \mathrm{Li}$ projectile, trials were also performed with a flat real potential not obeying the dispersion relations. In order to systematically probe the optical potential, the analysis of all data was carried out following the same standard procedure which can be described by the following main steps:

1) Draw a straight line to define the imaginary potential above the Coulomb barrier, consistent with previous experimental data extracted via an optical-potential analysis of angular distribution data 


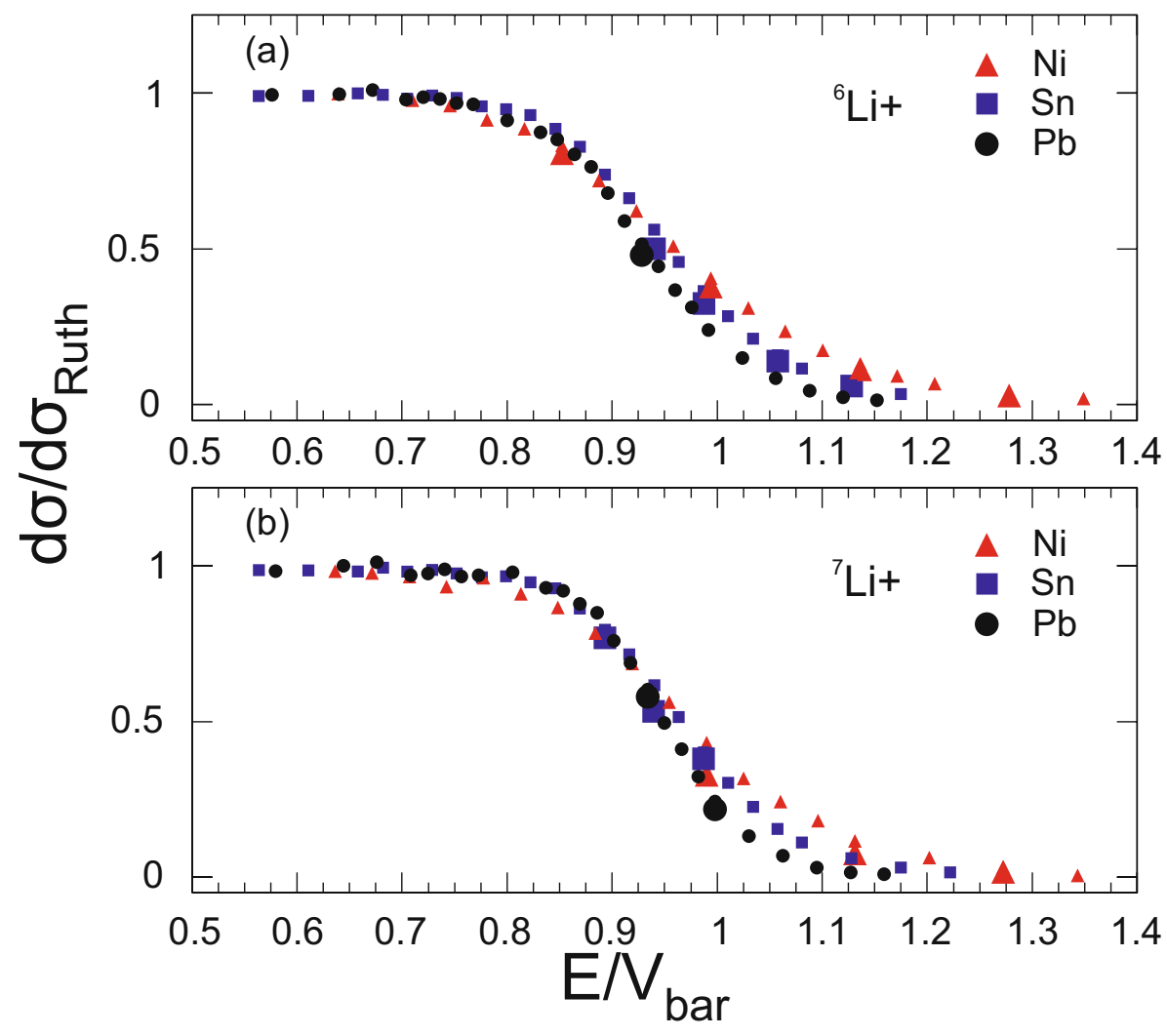

Fig. 1. (Color online) Excitation functions of average cross-sections between $160^{\circ}$ and $170^{\circ}$ (a) for the systems ${ }^{6} \mathrm{Li}+{ }^{58} \mathrm{Ni}$, ${ }^{6} \mathrm{Li}+{ }^{120} \mathrm{Sn},{ }^{6} \mathrm{Li}+{ }^{208} \mathrm{~Pb}$ and (b) for the systems ${ }^{7} \mathrm{Li}+{ }^{58} \mathrm{Ni},{ }^{7} \mathrm{Li}+{ }^{120} \mathrm{Sn},{ }^{7} \mathrm{Li}+{ }^{208} \mathrm{~Pb}$. The error is included in the size of the data points and did not exceed $6 \%$. Large data points indicate previous measurements taken from full angular distributions [31-34].

2) Define the energy point where a second line should be drawn with positive or negative slope. This second line is drawn with a certain slope and its start point is changed from higher to lower energies.

3) Define the slope of the second line which is drawn down to very low energies close to zero, changing it from negative slopes (conventional threshold anomaly) to more positive ones.

4) Iterate between points 2 and 3 till the best reduced $\chi^{2}$ is obtained between the calculated and experimentally determined barrier distributions.

5) Define the last energy point, where the imaginary potential drops to zero, changing it from higher to lower energies up to a point beyond which the fit is not any more sensitive to potential changes.

6) (If the final fit is not satisfactory.) Iterate between 1 to 5 changing the height of the first flat line describing the imaginary potential at energies above the Coulomb barrier.

\section{$3.1^{6,7} \mathrm{Li}+{ }^{58} \mathrm{Ni}$}

Elastic scattering angular distribution measurements for the ${ }^{6} \mathrm{Li}$ system have been presented before by Pfeiffer, Speth, Bethge [31] for near-barrier energies (experimental data A) and by E.F. Aguilera et al. [32] for sub-barrier energies (experimental data B). The data A were reanalyzed recently by Biswas et al. [35] in a double-folding BDM3Y1 framework as well as with a Woods Saxon potential. The energy dependence obtained for the imaginary part of the optical potential shows a steep increasing trend with decreasing energy at the barrier corresponding to a bump in the real part appearing at $E=0.8 V_{b a r}$, obeying a dispersion relation. The data $\mathrm{A}+\mathrm{B}$ were reanalyzed recently by A. Gómez Camacho et al. [36], with the doublefolding SPP interaction. In this case a rather flat energy dependence of the imaginary potential is predicted, which continues well below sub-barrier energies and finally drops to zero at $\sim 0.65 V_{b a r}$. The rather large errors of the fitting procedure, as expected in this energy region, may also allow a description of the imaginary potential consistent with a small increasing trend as the energy is decreasing. Both trends, the flat and the increasing one are accompanied by a peak in the real potential, located approximately at $E=0.6 V_{b a r}$. The data $\mathrm{A}+\mathrm{B}$ were also reanalyzed in the present study in our standard BDM3Y1 framework [24]. The uncertainty in all data points was considered to be $10 \%$. Our results for the potential are presented in fig. $2 \mathrm{~b}$ and $\mathrm{c}$ for the real and imaginary part of the optical potential, respectively, and are denoted with triangles. These results are compatible with those obtained by Gómez Camacho et al. [36], presenting a rather flat behavior and not the trend proposed in [35]. 


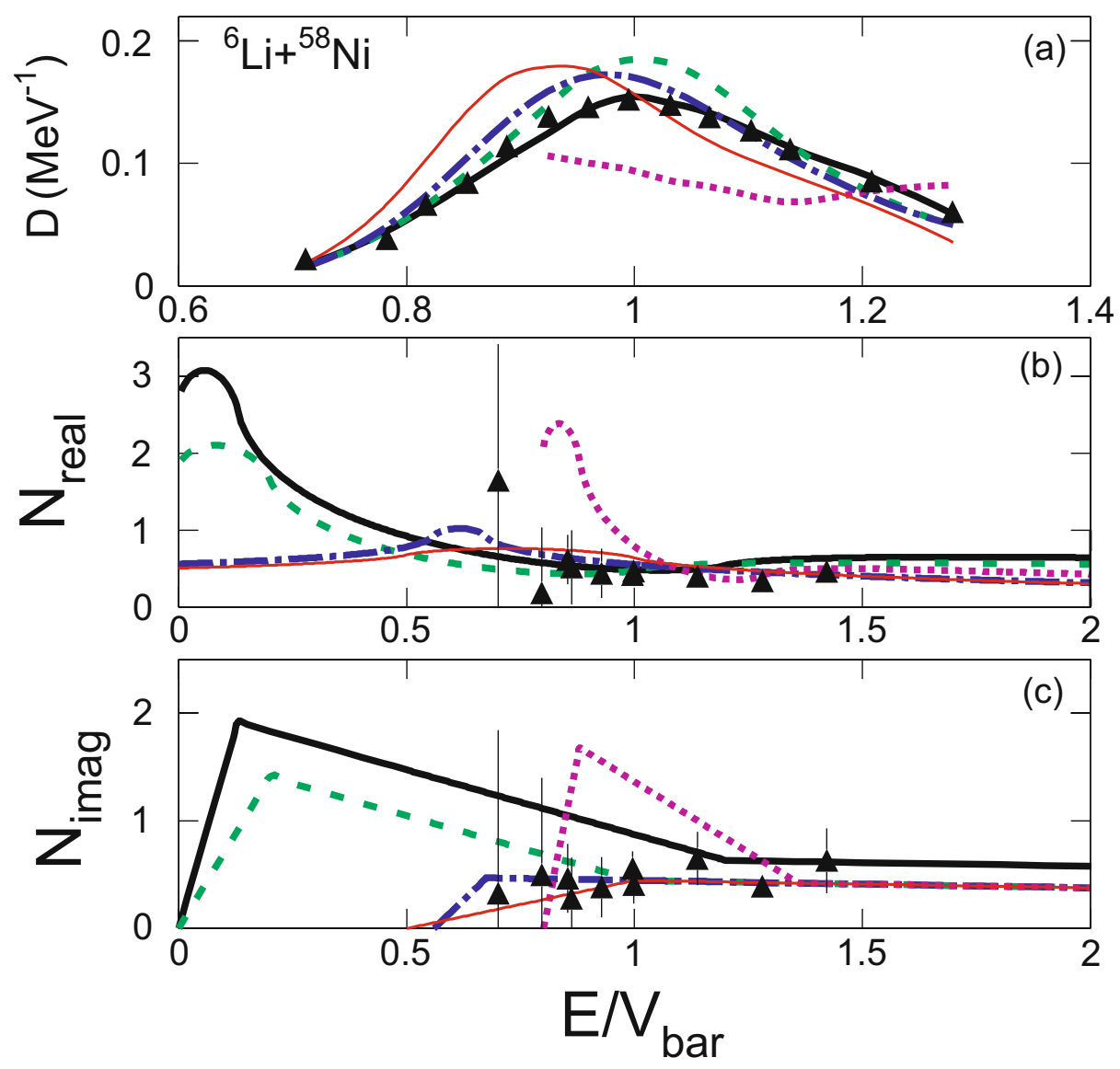

Fig. 2. (Color online) Barrier distributions for ${ }^{6} \mathrm{Li}+{ }^{58} \mathrm{Ni}$, obtained in this work, appear in panel a and are denoted with triangles. The error is included in the size of the data points and did not exceed in most of the cases $5 \%$. Calculations are also presented adopting the trial potentials shown in panels $\mathrm{b}$ and $\mathrm{c}$ for the real and imaginary part of the optical potential, respectively, following the same notation. Thick solid line (black line) corresponds to the best potential. The thin solid line (red line) corresponds to potential with a traditional threshold anomaly, the dot-dashed line (blue line) with the suggested potential by Camacho et al., the dotted line (purple line) with the potential suggested by Biswas et al. and the dashed line (green line) shows simply one of the many trials. In panels $b$ and $c$, with triangles are denoted the potentials obtained in the present study from fits to previous angular distribution measurements [31,32].

We will demonstrate now how the backscattering technique will reduce the ambiguity of the energy dependence of the potential as presented above, and further on, how it can provide the means for an accurate determination of the potential to very low sub-barrier energies.

In our usual approach [8,9], we start with various trial imaginary potentials, shown in fig. $2 \mathrm{c}$, by using as a guide the elastic scattering data existing at the higher energies. We commence the procedure, drawing a flat line from the higher-to-lower energies, and a second line describing the drop of the potential to zero at an energy close to the Coulomb barrier (thin solid line, red line). This is the conventional imaginary potential which appears in the threshold anomaly of well-bound nuclei (thin solid line, red line). We then continue with a similar trial potential, suggested by Gómez Camacho et al. [36] or suggested by the present reanalysis of the previous elastic scattering data (dotted dashed line, blue line), described also by a flat line but which continues to very low sub-barrier energies, dropping to zero at $E=0.65 V_{b a r}$. Finally, we continue with a third trial potential as suggested by the analysis of Biswas et al. [35], denoted by a dotted line purple line (a flat part accompanied by a line of positive slope and then a line with negative slope, dropping to zero at the barrier). The corresponding real parts in all three cases are calculated via dispersion relations [37]. Subsequently adopting such optical potentials, elastic scattering cross-sections are calculated for the backward angles $\theta_{l a b}=160^{\circ}$ and $170^{\circ}$, an average cross-section is extracted and used for calculating barrier distributions. The results are presented in fig. $2 \mathrm{a}$ with the same notation as the potentials of fig. $2 \mathrm{~b}$ and $\mathrm{c}$. It is obvious that the above optical potentials cannot reproduce the backscattering results of the barrier distribution. After several other trials, a "best" optical potential is adopted (thick solid line) as the one for which the predicted barrier distribution shows the best consistency with the measured one. It should be noted that several other trials, not all of them shown in the figure, were attempted to obtain the best energy point at which the imaginary potential starts rising as the energy is decreasing and also 


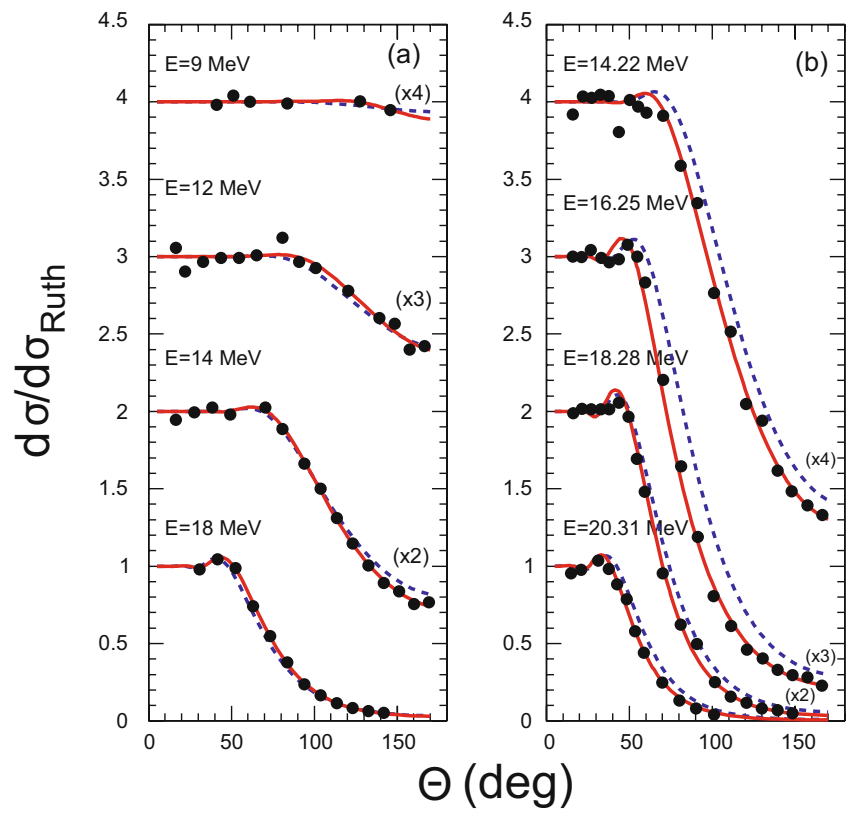

Fig. 3. (Color online) Previous elastic scattering data [32] are compared with best fits into a BDM3Y1 optical-model analysis (solid line, red line) and predictions obtained via optical potentials determined via the backscattering technique (dashed line, blue line) for the systems (a) ${ }^{6} \mathrm{Li}+{ }^{58} \mathrm{Ni}$ and (b) ${ }^{7} \mathrm{Li}+{ }^{58} \mathrm{Ni}$.

for the energy point where it starts dropping to zero. Some trials were also devoted to the height of the flat part of the line describing the imaginary potential at the higher energies.

Finally, in fig. 3a we present previous experimental data (data B) of elastic scattering angular distributions at some near barrier energies together with the best fits, obtained in the present reanalysis of these data in our usual BDM3Y1 framework. In the same figure we present predicted cross-sections according to the proposed potentials obtained via the backscattering barrier distribution technique and the agreement at most of the energies is satisfactory.

Subsequently, we carried out a similar procedure for ${ }^{7} \mathrm{Li}+{ }^{58} \mathrm{Ni}$. Some trial potentials and the "best" one are shown in fig. $4 \mathrm{~b}$ and $\mathrm{c}$ the real and imaginary part, respectively, while the corresponding barrier distribution predictions are compared with the data in fig. 4a. For ease of comparison the same notation of lines is used in these figures. In more detail, taking into account our previous work on ${ }^{7} \mathrm{Li}+{ }^{28} \mathrm{Si}$, we have considered an optical potential with the real part in one case described by a line deduced from the imaginary potential via a dispersion relation (thick lines) and in the second case described simply by a flat line not obeying the dispersion theory (thin lines). We start with a trial imaginary potential which fits few data extracted from the reanalysis of elastic scattering results [31] via our standard BDM3Y1+ECIS procedure. The barrier distributions, obtained either with a potential obeying the dispersion relation (thick dot-dashed line, blue line) or with one that does not (thin dot-dashed line, blue line), are not compatible with the backscatter- ing data. Subsequently, we proceeded with trials reducing substantially the flat part of the line describing the higher energy imaginary potential, while changing gradually the slope of the decreasing low-energy part. The best optical potential obtained gives almost equally good predictions for the barrier distribution data (thick solid line with dispersion, thin solid line without dispersion-black lines) with slightly different reduced $\chi^{2}$ 's (with dispersion $\chi^{2}=2.7$, without dispersion $\chi^{2}=1.2$ ). Note the "step" in the imaginary potential in all trials, is necessary to absorb flux from the elastic channel. Finally, in fig. 3b we present previous experimental data (data B) of elastic scattering angular distributions at some near-barrier energies together with the best fits, obtained in the present reanalysis of these data in our usual BDM3Y1 framework. In the same figure we present predicted cross-sections according to the proposed potentials obtained via the backscattering barrier distribution technique. In this case the agreement at the lower energies, between predictions of backscattering and angular distribution data, is adequately good only at the extremes of a 10\% error. Despite several trial attempts it was impossible to find a potential describing both sets of data indicating that new angular distribution measurements may be necessary. In principle this disagreement does not exclude problems in the present barrier distribution data. Taking into account that the present data were determined with small errors and that data on other targets do not present similar large inconsistencies, with some caution we give more confidence to the present data.

\section{$3.2{ }^{6,7} \mathrm{Li}+{ }^{116,120} \mathrm{Sn}$ and ${ }^{6,7} \mathrm{Li}+{ }^{208} \mathrm{~Pb}$}

A similar analysis, as in the previous subsection, was applied for our results on $\mathrm{Sn}$ and $\mathrm{Pb}$ targets and the various trials for obtaining the optical potentials taking as a reference point the barrier distribution, are given in figs. 5 to 8. Results on the experimental barrier distributions of ${ }^{7} \mathrm{Li}+{ }^{116} \mathrm{Sn}$ and ${ }^{7} \mathrm{Li}+{ }^{120} \mathrm{Sn}$ are presented in fig. 9. These results indicate that there is no difference between the two isotopes at least in the context of the barrier distribution method and no further consideration of ${ }^{116} \mathrm{Sn}$ was adopted.

\subsection{Comparison of potentials}

After completing the study of all the systems, three with ${ }^{6} \mathrm{Li}$ and three with ${ }^{7} \mathrm{Li}$, we compare, in figs. 10 and 11, the optical potentials obtained via the backscattering technique. For reasons of completeness we present in the same figure the previous results on ${ }^{6,7} \mathrm{Li}+{ }^{28} \mathrm{Si}[8,9]$. The following conclusions can be drawn.

For ${ }^{6} \mathrm{Li}$, the imaginary potential presents an increasing trend from higher to lower energies. This rising part has the largest slope for the heavier targets and the smallest slope for the lighter ones. Qualitatively, this may be interpreted to be connected with the breakup channel which is expected to be larger for the heavier targets (larger $Z$ ) 


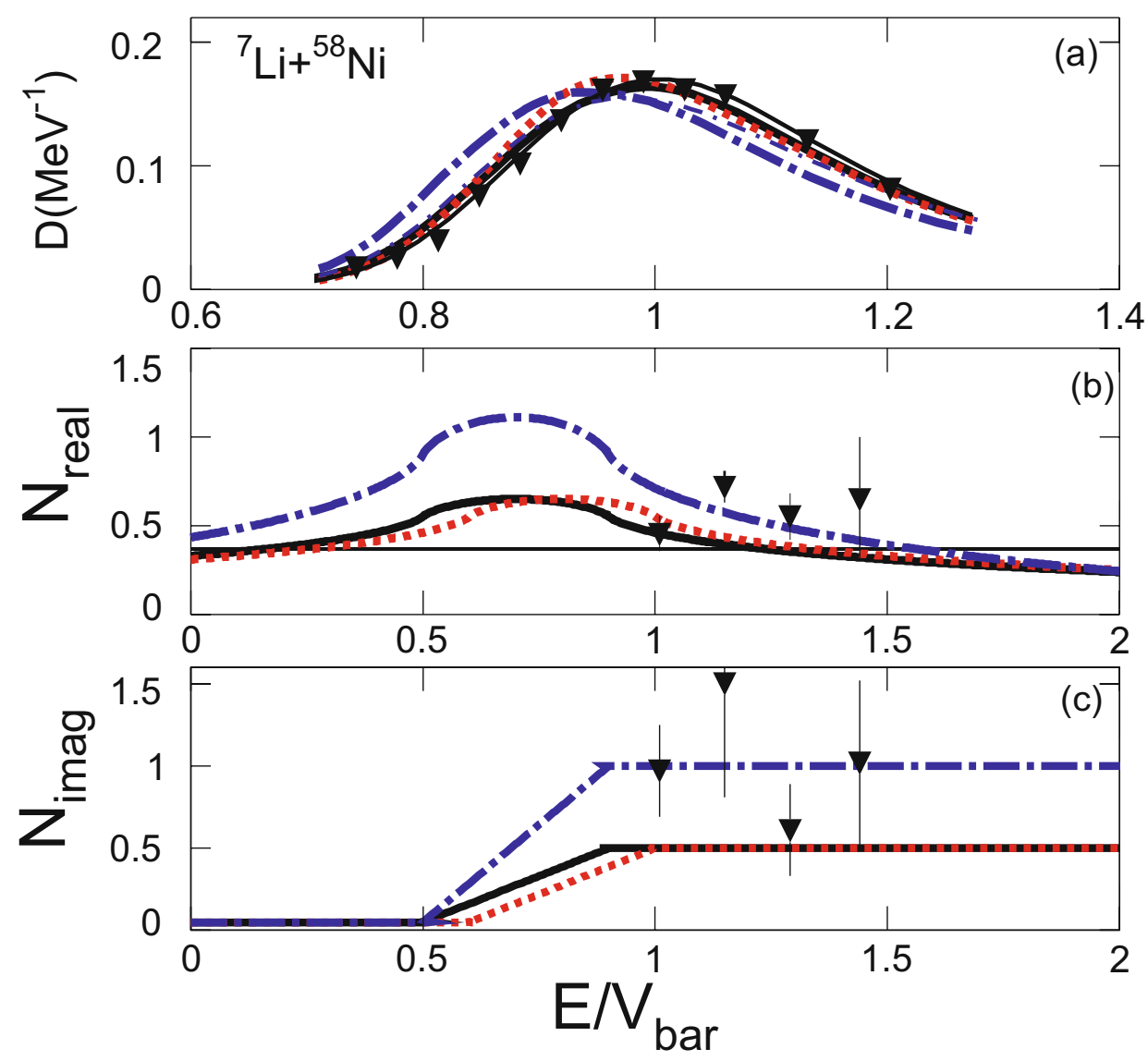

Fig. 4. (Color online) Barrier distributions for ${ }^{7} \mathrm{Li}+{ }^{58} \mathrm{Ni}$, obtained in this work, appear in panel a and are denoted with triangles. The error is included in the size of the data points and did not exceed in most of the cases $5 \%$. Various lines correspond to calculations with the trial potentials shown in panels b and $\mathrm{c}$ for the real and imaginary part of the optical potential, respectively, following the same notation. The solid black line represent calculation with the "best potential" and two other trial optical potentials are represented with the dot-dashed line (blue line) and dotted line (red line). The thick lines represent calculations adopting a potential obeying a dispersive relation, while the thin lines adopting a potential without a dispersive relation. In panels $\mathrm{b}$ and $\mathrm{c}$, with triangles are denoted the potentials obtained in the present study from fits to previous angular distribution measurements [31].

and smaller for the lighter ones (smaller $Z$ ) without excluding transfer contributions. Another interesting point is that the energy where the imaginary potential drops to zero is not located at the barrier but at sub-barrier energies, in contrast with the behaviour of well-bound nuclei. It should be underlined however that the low energy where the imaginary potential drops to zero for the nickel and silicon targets near $E / V_{\text {bar }} \sim 0.2$ raises some questions about the validity of the dispersion relation. It is hardly credible that at this energy such an enormous real dynamical polarization potential almost two times the bare one can be generated. Also it is difficult to think of a physical process which generates such large amounts of absorption.

For the ${ }^{7} \mathrm{Li}$ case, a possible break down of the dispersion relation was even more intensified. The problem which has arisen was that the backscattering barrier distributions were well predicted by a potential (polarization potential) obeying the dispersion relation but also by one that does not with similar $\chi$ 's for the two solutions, although the smallest $\chi^{2}$ distribution always occurs for the second scenario. If we take into account the case where a dispersion relation holds, then the imaginary potential can be described by a flat line which progresses to very low energies, with a drop to zero between $E / V_{\text {bar }} \sim 0.5$ to 0.9 . If we finally assume that the dispersion relation does not hold then the potential starts dropping to zero between $E / V_{\text {bar }} \sim 0.8$ to 1.2 and then continues with a flat step to very low sub-barrier energies. Transfer measurements may be necessary to indicate the correct energy point where the imaginary potential drops to zero and therefore to decide between the two scenarios. In either case the optical potential behaviour obtained for the ${ }^{7} \mathrm{Li}$ projectile contrasts with the trend for ${ }^{6} \mathrm{Li}$, where we observe a rising behaviour towards the lower energies. On the other hand, for both weakly bound projectiles the common point is a loss of flux from the elastic channel persisting to very low energies. This is reflected in the imaginary potential which continues to very low sub-barrier energies either with an increasing trend towards lower energies in the case of ${ }^{6} \mathrm{Li}$, or with a flat line or/and a small "step" potential in the case of ${ }^{7} \mathrm{Li}$. As was also noticed in the ${ }^{6} \mathrm{Li}$ case, the proposed potentials do not follow any systematics due to the 


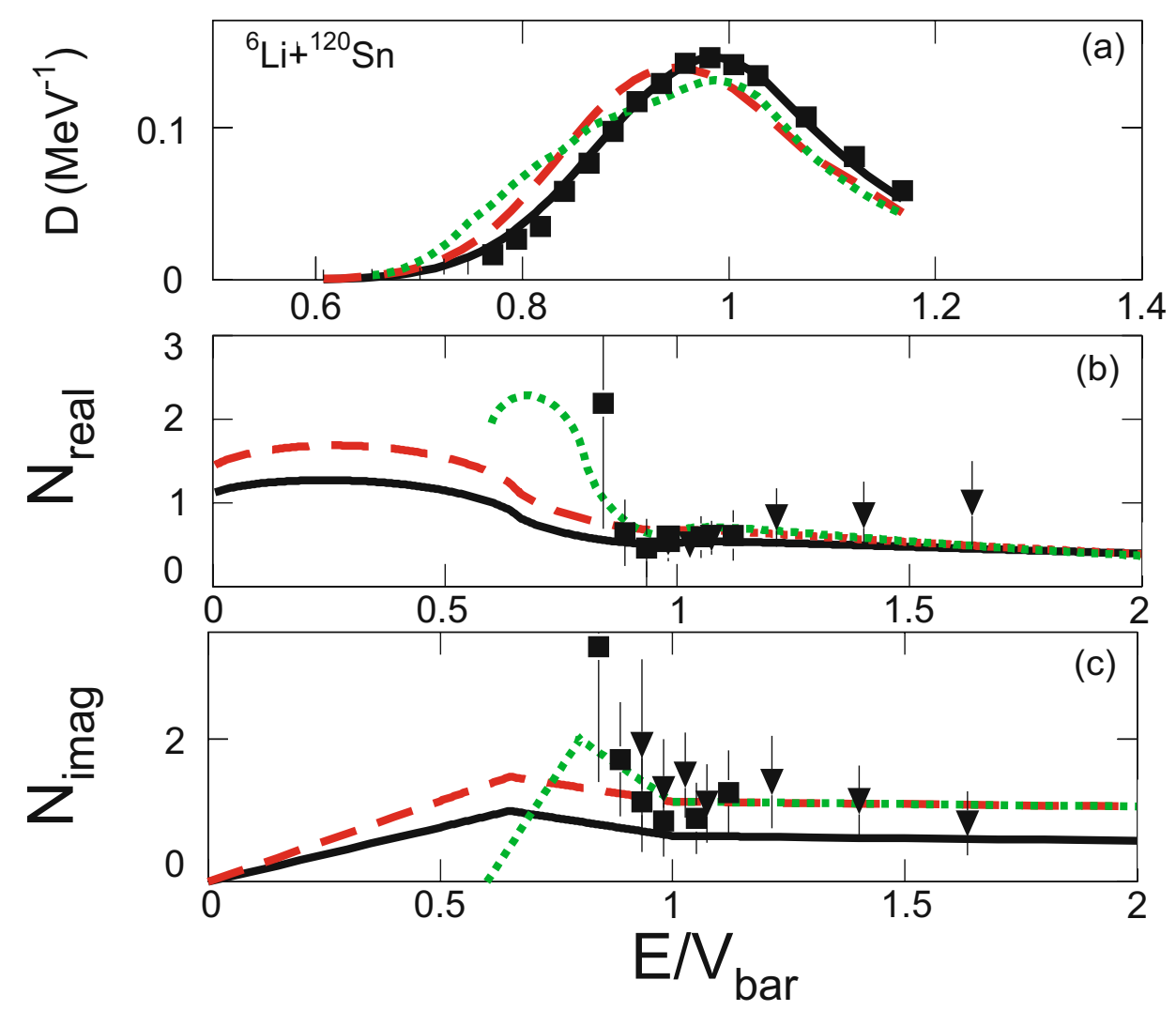

Fig. 5. (Color online) Barrier distributions for ${ }^{6} \mathrm{Li}+{ }^{120} \mathrm{Sn}$, obtained in this work, appear in panel a and are denoted with solid boxes. The error is included in the size of the data points and did not exceed in most of the cases $5 \%$. Calculations are also presented adopting the trial potentials shown in panels $\mathrm{b}$ and $\mathrm{c}$ for the real and imaginary part of the optical potential, respectively, following the same notation. Thick solid line (black line) correspond to the best potential and the other two lines, the dashed line (red line) and the dotted line (green line) show simply some more trials. In panels b and c, with the solid boxes are denoted the potentials obtained from fits to previous angular distribution measurements for the system ${ }^{6} \mathrm{Li}+{ }^{118} \mathrm{Sn}[31]$ and with triangles the potentials for the system ${ }^{6} \mathrm{Li}+{ }^{116} \mathrm{Sn}[34]$.

different reaction mechanisms involved and are unique for each target. This finding is in accordance with the results on polarisation potentials studied by Lubian et al. [6]. The authors claim that the net result of polarisation potentials describing transfer and breakup may vary from system to system depending on the charge of the target and consequently on the relative strength of the Coulomb and nuclear potentials. Unfortunately this fact prohibits a systematic description of the potential as a function of mass and energy for weakly bound nuclei.

\subsection{Fusion barriers}

Fusion barriers extracted from the barrier distributions are shown in tables 1 and 2, and are compared with theoretical values extracted from phenomenological parametrisations [38]. Other details of the barrier distributions such as the widths and heights are also included in the same tables.

From the systematics of the barrier distributions we can draw the following conclusions: The measured barriers agree very well with values calculated via the ChristensonWinther parametrisations [38]. For the lighter targets the agreement is excellent. The widths of the barrier distributions are larger for the heavier targets, indicating stronger quantal effects, generated by couplings to various reaction channels. For ${ }^{6} \mathrm{Li}$ this is reflected in the increasing trend of the imaginary part of the optical potential (line with a positive slope). The heavier the target the larger the slope. On the other hand the energy point where the imaginary potential drops to zero is located at much lower energies for the lighter targets than for the heavier ones. Comparing the barrier widths of ${ }^{6} \mathrm{Li}$ and ${ }^{7} \mathrm{Li}$ we see that the widths are larger for the first projectile. This fact is also reflected in the imaginary potential which rises at sub-barrier energies for ${ }^{6} \mathrm{Li}$ but is flat for ${ }^{7} \mathrm{Li}$. In both cases the widths are much larger than those observed for well-bound projectiles, in accordance with the proposed potentials for weakly bound ones, where the imaginary part persists to very low energies below the barrier. The heights of the barrier distributions are systematically larger for the lower mass targets, possibly indicating a stronger surface penetrability for the heavier ones. 


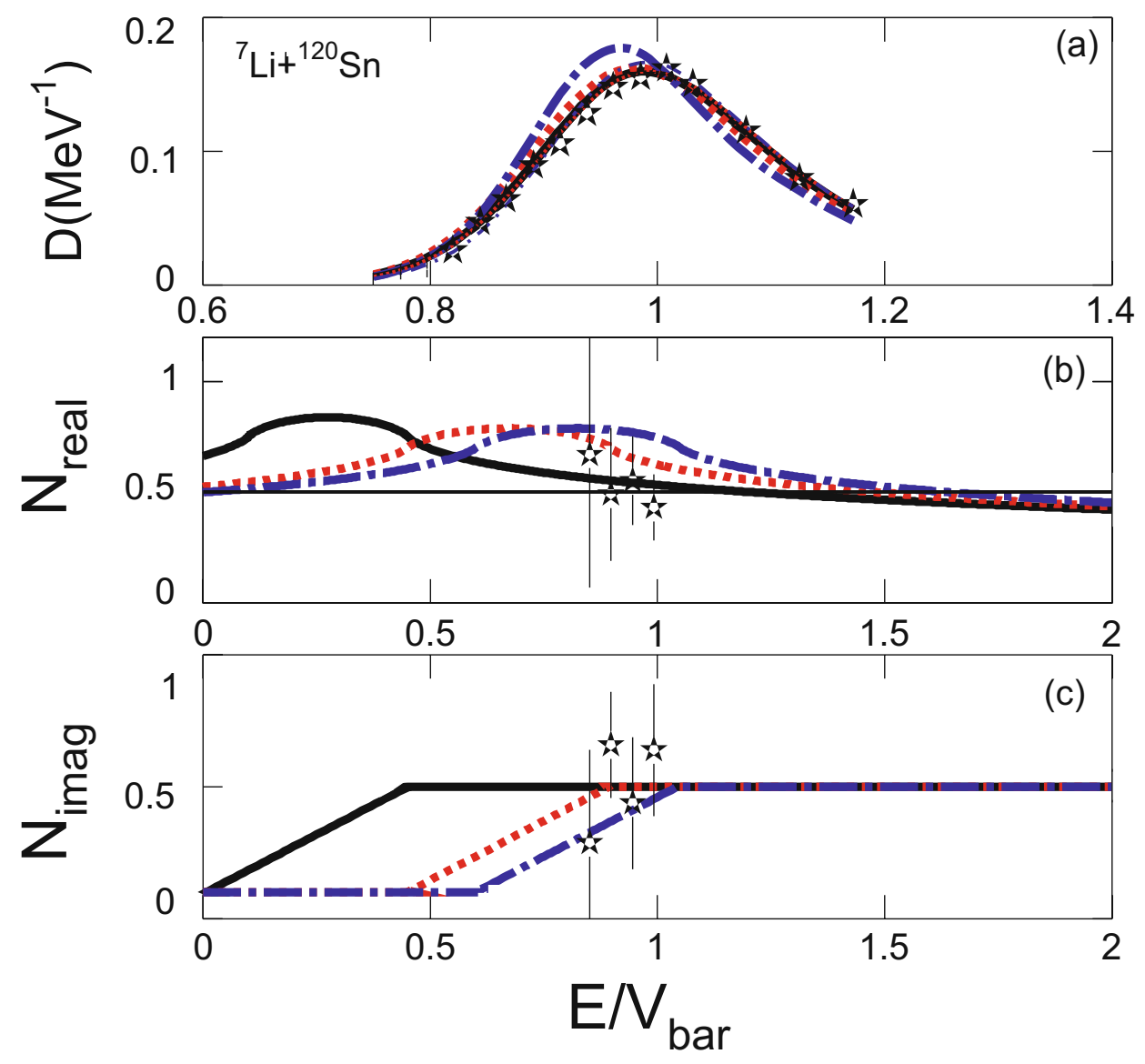

Fig. 6. (Color online) Barrier distributions for ${ }^{7} \mathrm{Li}+{ }^{120} \mathrm{Sn}$ obtained in this work, appear in panel a and are denoted with stars. The error is included in the size of the data points and did not exceed in most of the cases $5 \%$. Various lines correspond to calculations with the trial potentials shown in panels $\mathrm{b}$ and $\mathrm{c}$ for the real and imaginary part of the optical potential, respectively, following the same notation. The solid black line represent calculation with the "best potential" and two other trial optical potentials are represented with the dotted dashed line (blue line) and dotted line (red line). The thick lines represent calculations adopting a potential obeying a dispersive relation, while the thin lines adopting a potential without a dispersive relation. Thin lines are not well distinguishable from the thick solid black line since we obtain equally good fits when the real potential does not obey the dispersion relation. In panels b and c, with stars are denoted the potentials obtained in the present study from fits to previous angular distribution measurements [31].

\section{The reaction mechanisms}

In our previous work $[8,9]$ we showed by means of CDCC calculations that the breakup of ${ }^{6} \mathrm{Li}$ strongly affects the barrier distribution for the ${ }^{6} \mathrm{Li}+{ }^{28} \mathrm{Si}$ system, shifting it to slightly higher energies and reducing the peak height by at least $20 \%$ while simultaneously making the distribution more broad. We have also shown for ${ }^{6} \mathrm{Li}+{ }^{28} \mathrm{Si}$ in a full Coupled Reaction Channel (CRC) calculation that although transfer is a strong channel, coupling to it has a minor effect on the barrier distribution. On the other hand we have shown that the breakup of ${ }^{7} \mathrm{Li}$ does not affect the barrier distribution and coupling to the continuum seems unimportant.

In this work we have performed CDCC calculations for ${ }^{6,7} \mathrm{Li}+{ }^{58} \mathrm{Ni},{ }^{6,7} \mathrm{Li}+{ }^{120} \mathrm{Sn}$ and ${ }^{6,7} \mathrm{Li}+{ }^{208} \mathrm{~Pb}$, following closely those presented in ref. [19]. It was assumed that the nucleus ${ }^{6} \mathrm{Li}\left({ }^{7} \mathrm{Li}\right)$ has a two-body $\alpha+d(\alpha+t)$ cluster structure. Couplings between resonant and non-resonant cluster states corresponding to $\alpha-d(\alpha-t)$ relative orbital angular momentum $L=0,1,2(L=0,1,3)$ were included. For ${ }^{7} \mathrm{Li}$ excitation of the first-excited-state and ground-state reorientation were taken into account. The continuum above the ${ }^{6} \mathrm{Li} \rightarrow \alpha+d\left({ }^{7} \mathrm{Li} \rightarrow \alpha+t\right)$ breakup threshold was discretized into momentum bins. The width of most of the bins was set to $\Delta k=0.26 \mathrm{fm}^{-1}$ for ${ }^{6} \mathrm{Li}$ and to $\Delta k=0.25 \mathrm{fm}^{-1}$ for ${ }^{7} \mathrm{Li}$. In the presence of resonant states the binning schemes were suitably modified in order to avoid double counting. Upper limits of the continuum states were taken, e.g., for $13 \mathrm{MeV}{ }^{6,7} \mathrm{Li}+{ }^{28} \mathrm{Si}$ as 10.6 MeV and $9.3 \mathrm{MeV}$ correspondingly and reduced for the lower energies according to the appropriate value of $E_{c . m}$. of the system. All the diagonal and coupling potentials were generated from empirical $\alpha+$ Target, $d+$ Target and $t+$ Target optical model potentials derived from elastic scattering experiments [39-43]. 


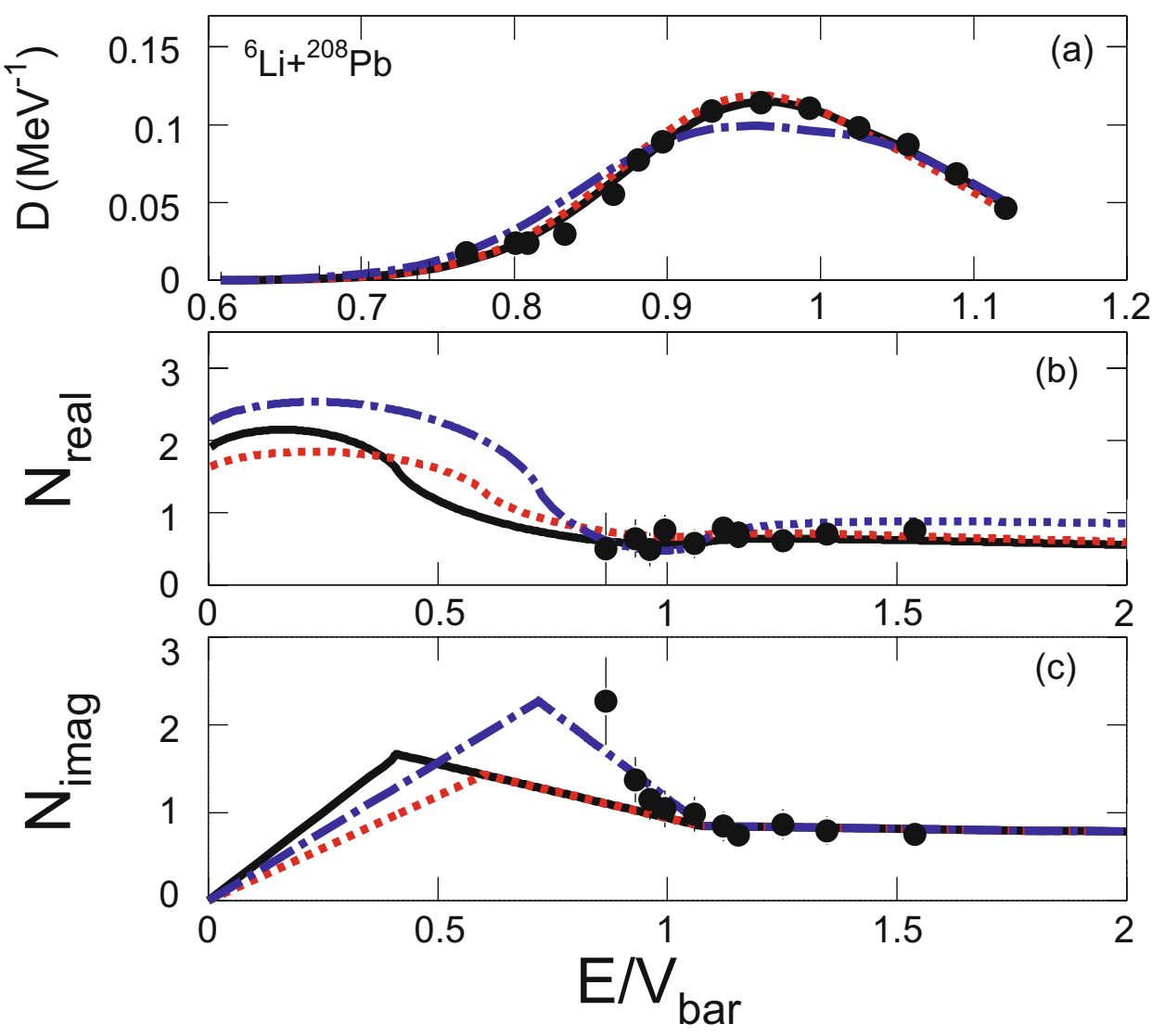

Fig. 7. (Color online) Barrier distributions for ${ }^{6} \mathrm{Li}+{ }^{208} \mathrm{~Pb}$ obtained in this work, appear in panel a denoted with solid circles. The error is included in the size of the data points and did not exceed in most of the cases $5 \%$. Calculations are also presented adopting the trial potentials shown in panels $\mathrm{b}$ and $\mathrm{c}$ for the real and imaginary part of the optical potential, respectively, following the same notation. Thick solid line (black line) correspond to the best potential. The dot-dashed line (blue line) is the one that follows the elastic scattering data while the dotted line (red line) shows simply one of the many trials. In panels $\mathrm{b}$ and $\mathrm{c}$, with the circles are denoted the potentials obtained in the present study from fits to previous angular distribution measurements [33].

Since in the backscattering measurements of ${ }^{7} \mathrm{Li}$ on the nickel and tin targets the inelastic part was not resolved from the elastic part the calculations for ${ }^{7} \mathrm{Li}$ represent the quasi-elastic scattering (elastic + excitation of ${ }^{7} \mathrm{Li}$ to its first excited state). The backscattering results are presented in figs. 12, 13 and 14 and are compared with the CDCC calculations. It is seen that for all cases coupling to the continuum improves the description of the barrier distributions. The effect for the ${ }^{6} \mathrm{Li}$ cases, as noted in our previous study with a silicon target [8], is very strong, shifting the barrier to higher energies and reducing the barrier distribution height. For the ${ }^{7} \mathrm{Li}$ cases the effect is much smaller. With no obvious reason a disagreement between the calculation and the data is noted only for ${ }^{7} \mathrm{Li}+{ }^{120} \mathrm{Sn}$. A full CRC calculation taking into account the effect of transfer is beyond the scope of the present work but it may give a solution to the problem.

\section{Conclusions and discussion}

Elastic backscattering excitation functions and the corresponding barrier distributions were measured for ${ }^{6,7} \mathrm{Li}$ projectiles incident on ${ }^{58} \mathrm{Ni},{ }^{116,120} \mathrm{Sn}$ and ${ }^{208} \mathrm{~Pb}$ targets. The present results were combined with previous ones on ${ }^{28} \mathrm{Si}$ and the optical potential based on dispersion relations was probed in a systematic way at energies close to and well below the barrier at deep sub-barrier energies.

Although a lack of systematic behaviour prohibits the deduction of a global optical potential for weakly bound projectiles at sub-barrier energies, considerable conclusions can be drawn and may be summarized as follows:

1) The backscattering technique is a valuable tool for predicting in a complementary way, the optical potential at sub-barrier energies. While it is at the moment the only tool for predicting the potential to some extend at energies below the barrier, where the conventional angular distribution technique is not sensitive to the nuclear potential, for providing detailed information on this issue, extensive and precise experimental data of various kinds are required. Unfortunately at these low energies the measurement of reaction cross-sections of any type is a very difficult task. 


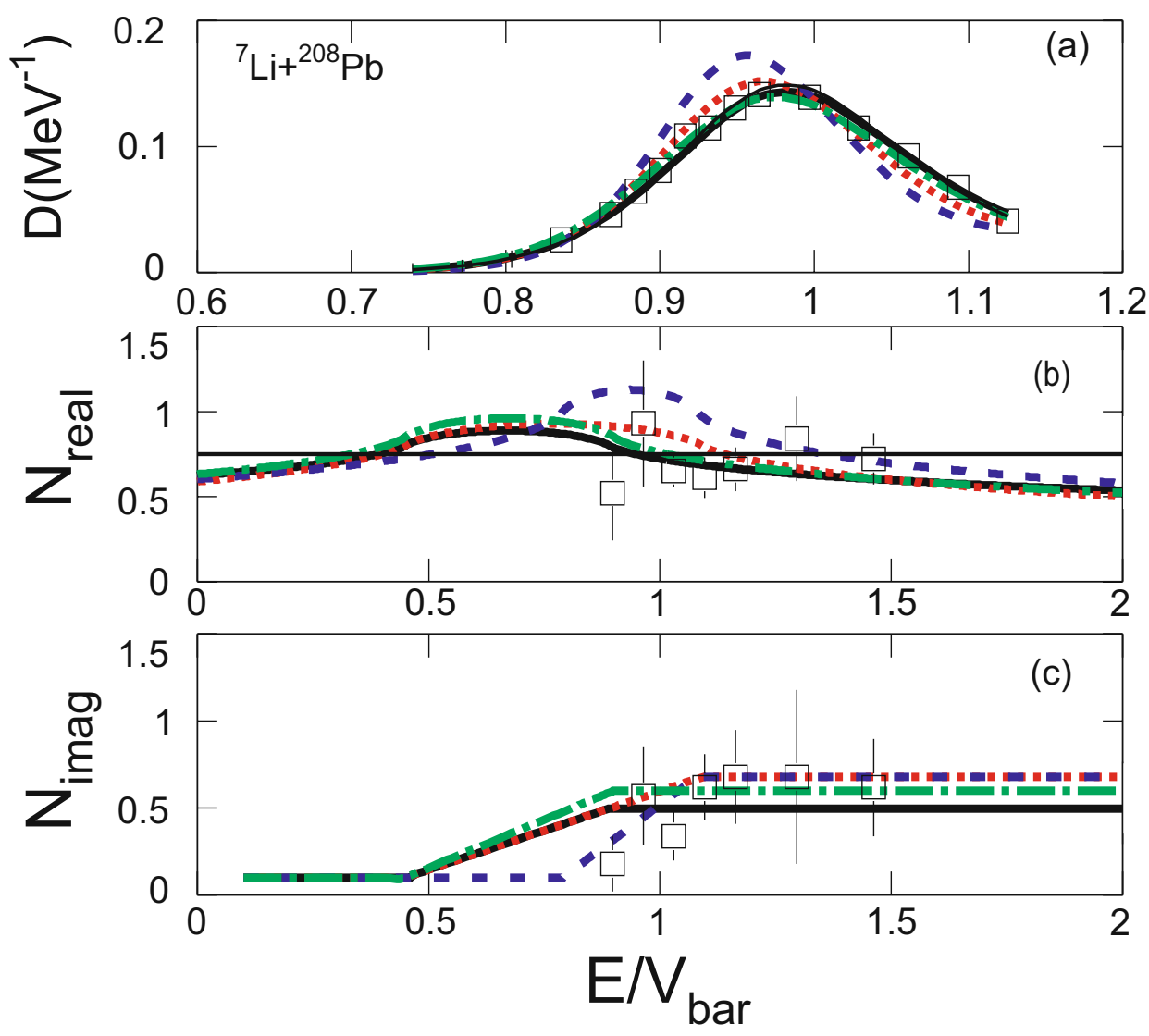

Fig. 8. (Color online) Barrier distributions for ${ }^{7} \mathrm{Li}+{ }^{208} \mathrm{~Pb}$ obtained in this work, appear in panel a denoted with empty boxes. The error is included in the size of the data points and did not exceed in most of the cases $5 \%$. Various lines correspond to calculations with the trial potentials shown in panels b and $\mathrm{c}$ for the real and imaginary part of the optical potential, respectively, following the same notation. The solid black line represent calculation with the "best potential" and some other trial optical potentials are represented with the dot-dashed line (green line), the dotted line (red line) and the dashed line (blue line). The thick lines represent calculations adopting a potential obeying a dispersive relation, while the thin lines adopting a potential without a dispersive relation. In panels $\mathrm{b}$ and $\mathrm{c}$, with boxes are denoted the potentials obtained in the present study from fits to previous angular distribution measurements [33].

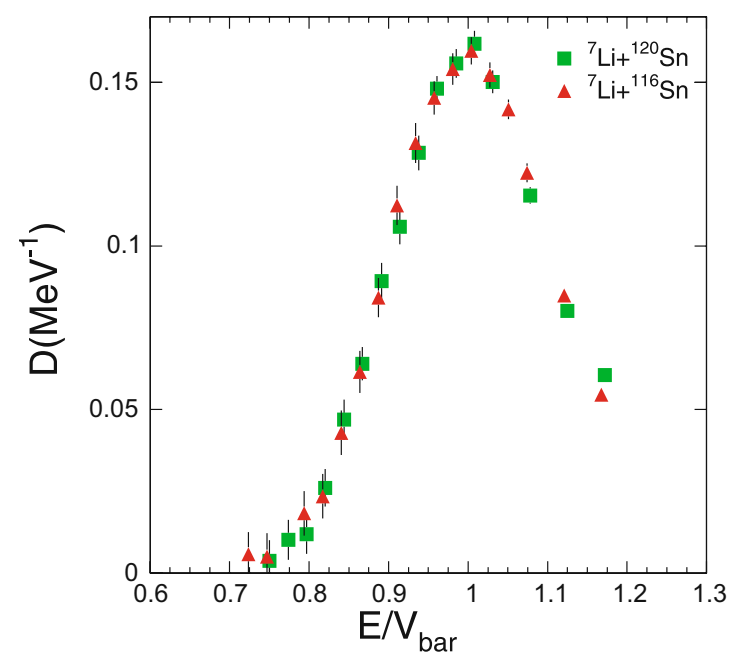

Fig. 9. (Color online) Comparison of barrier distributions for ${ }^{7} \mathrm{Li}+{ }^{116} \mathrm{Sn}$ and ${ }^{7} \mathrm{Li}+{ }^{120} \mathrm{Sn}$, denoted with solid triangles and boxes, respectively.
2) For weakly bound nuclei the imaginary potential persists either with an increasing trend $\left({ }^{6} \mathrm{Li}\right)$ or a flat behaviour $\left({ }^{7} \mathrm{Li}\right)$ to sub-barrier energies near $E / V_{\text {bar }}=$ 0.5 , in contrast with well-bound nuclei where the loss of flux from the elastic channel stops around the Coulomb barrier. For some targets the drop occurs at very low energies, $\sim E / V_{b a r}=0.2$, associated with a very large real part, unexpected under physical conditions. This fact puts on stake the validity of the dispersion relation.

3) For ${ }^{6} \mathrm{Li}$ the rising part has the largest slope for the heavier targets and the smallest slope for the lighter ones, possibly indicating in a qualitative interpretation that the competition between breakup and transfer or some compound procedure at energies at the barrier is in favour of breakup. However, as we proceed to lower sub-barrier energies, the competition of the various channels is not clear but only under a complete set of reaction cross-section measurements. 

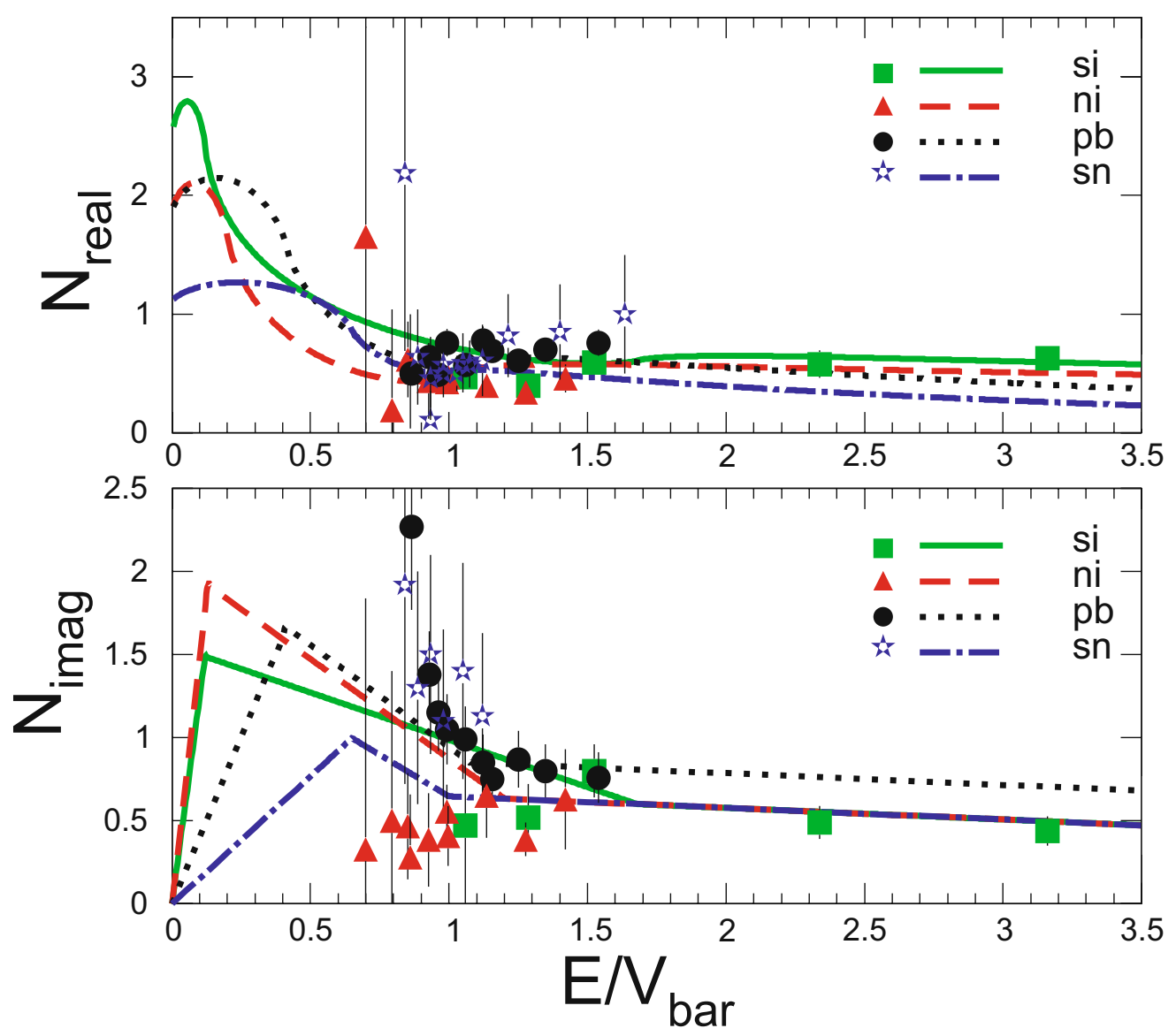

Fig. 10. (Color online) Energy dependence of the optical potentials extracted from the present barrier distribution analysis of ${ }^{6} \mathrm{Li}$ on nickel, tin and lead targets. Previous results [8] for a silicon target are also presented. Results obtained from a conventional reanalysis of elastic scattering distributions on various energies previously measured [18,31-34] are compared with them. For the online reader it should be mentioned that symbols and lines of the same colour refer to the same colliding system.

4) For ${ }^{7} \mathrm{Li}$, while the backscattering technique also proves to be a valuable tool for predicting the potential at very low sub-barrier energies, it is not sensitive enough to distinguish between a potential obeying the dispersion relation and one which does not, although the trend is in favour of the second scenario. Studies including other targets are necessary to pin down this problem.

In principle, it can be shown that the optical potential that comes out of Feshbach theory must formally satisfy a dispersion relation. However, this is a theoretical construct and therefore not the same as the empirical optical potential that we obtain from fits to elastic scattering data. Therefore, the empirical optical potential does not necessarily have to satisfy a dispersion relation [37]. In the present case, with caution we can say that this is observed as an absence of an energy dependence of the potential, an observation which can generate an important theoretical work. A possible explanation, although under theoretical investigation for the moment, is that this is due to the interference of the repulsive polarization potential due to the break-up process, with the attractive polarization potential generated by transfer reactions in- volving the bound excited state of ${ }^{7} \mathrm{Li}$. The strength of these two components of the polarization potential could be equal and of opposite sign one repulsive the other attractive and the net effect may be a non-energy-dependent optical potential. The presence or a possible absence of the so-called "break-up threshold anomaly" in the optical potential of the scattering of weakly bound nuclei is a question of paramount importance. It may challenge our current theoretical understanding of low energy reaction dynamics involving weakly bound nuclei and modify our current understanding of the genesis and production rate of some nuclei produced in stellar explosions.

We have been the first to experimentally demonstrate [19] that the "break-up threshold anomaly" although present in the case of elastic scattering including ${ }^{6} \mathrm{Li} \mathrm{pro-}$ jectiles was absent in the case of ${ }^{7} \mathrm{Li}$ nuclei. This experimental finding for a silicon target raised an important criticism and a consequent experimental and theoretical work. Since our first publications, the absence of the threshold anomaly in the case of ${ }^{7} \mathrm{Li}$, that is the absence of energy dependence of the real and imaginary part of the optical potential, has been reported in a number of experimental papers by other groups and for other mass regions as well and now reported in a more systematic way in this work. 

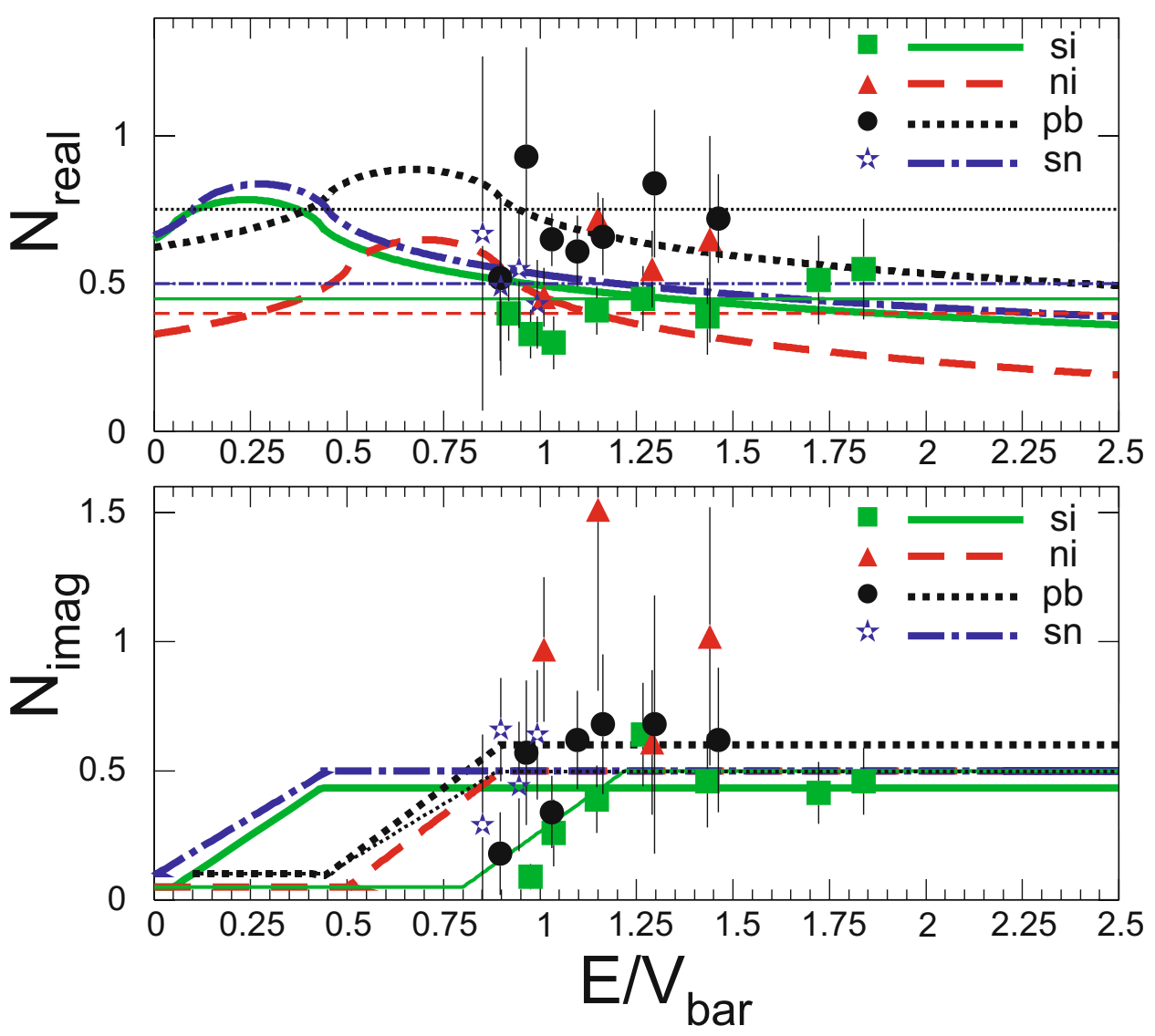

Fig. 11. (Color online) Energy dependence of the optical potentials extracted from the present barrier distribution analysis of ${ }^{7} \mathrm{Li}$ on nickel, tin and lead targets. Previous data [8] for a silicon target are also presented. Thin lines represent best potentials where the dispersion relation does not hold. Results obtained from a conventional reanalysis of elastic scattering distributions on various energies previously measured $[19,31,33]$ are compared with them. For the online reader it should be mentioned that symbols and lines of the same colour refer to the same colliding system.

Table 1. Details of barrier distributions for ${ }^{6} \mathrm{Li}$ on various targets. The obtained fusion barriers, $V_{b a r}{ }^{\text {meas. }}$, are compared with calculated values $V_{\text {bar }}{ }^{\text {calc. }}$, according to ChristensenWinther [38].

\begin{tabular}{ccccc}
\hline Target & $V_{\text {bar }}$ meas. & $V_{\text {bar }}{ }^{\text {calc. }}$ & Width & Height \\
\hline${ }^{28} \mathrm{Si}$ & 8.5 & 8.5 & 3.8 & 0.2 \\
${ }^{58} \mathrm{Ni}$ & 14.0 & 14.1 & 5.5 & 0.15 \\
${ }^{120} \mathrm{Sn}$ & 20.9 & 21.4 & 6.0 & 0.15 \\
${ }^{208} \mathrm{~Pb}$ & 30.0 & 31.2 & 7.5 & 0.11 \\
\hline
\end{tabular}

This result should initiate more studies including other targets and various techniques from the point of view of the potential and the reaction mechanism itself. Moreover in this study the validity of dispersion relation has also been questioned in the case of ${ }^{6} \mathrm{Li}$ projectiles.

From the point of view of the reaction mechanisms, via our CDCC calculations it became clear once more that couplings to the continuum are strong and important. This fact was also verified in similar work on samarium, thorium and lead in [20-23]. However, to obtain a full insight
Table 2. Details of barrier distributions for ${ }^{7} \mathrm{Li}$ on various targets. The obtained fusion barriers, $V_{b a r}{ }^{\text {meas. }}$, are compared with calculated values $V_{\text {bar }}{ }^{\text {calc. }}$, according to ChristensenWinther [38].

\begin{tabular}{ccccc}
\hline Target & $V_{\text {bar }}$ meas. & $V_{\text {bar }}$ calc. & Width & Height \\
\hline${ }^{28} \mathrm{Si}$ & 8.5 & 8.7 & 3.3 & 0.22 \\
${ }^{58} \mathrm{Ni}$ & 14.0 & 14.1 & 4.8 & 0.17 \\
${ }^{120} \mathrm{Sn}$ & 21.5 & 21.3 & 5.3 & 0.16 \\
${ }^{208} \mathrm{~Pb}$ & 30.3 & 31.1 & 5.6 & 0.14 \\
\hline
\end{tabular}

into the reaction mechanism and how it is related to the predicted optical potential more work from the experimental and theoretical points of view is necessary. This should include backscattering measurements from several targets, transfer and breakup measurements for the same targets - the latter as tracers of the predicted potentials - as well as full CRC calculations where transfer will be included simultaneously with breakup. The case of ${ }^{7} \mathrm{Li}+{ }^{120} \mathrm{Sn}$ remains open to a new CDCC calculation since at the moment our calculation fails to describe our data. 


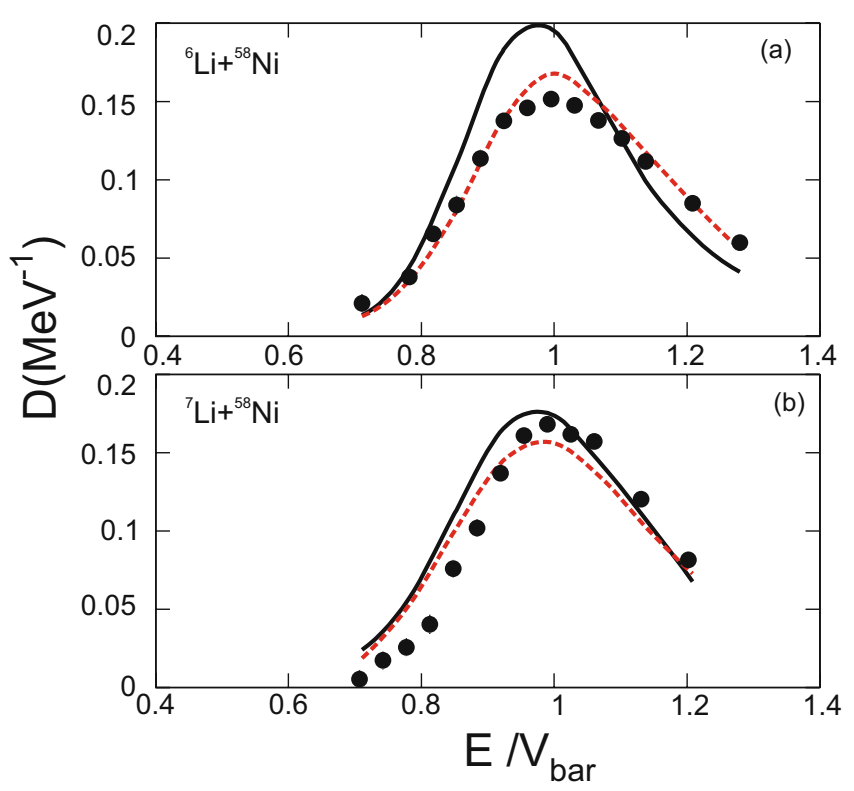

Fig. 12. (Color online) Experimental data of barrier distributions for (a) ${ }^{6} \mathrm{Li}+{ }^{58} \mathrm{Ni}$ and (b) ${ }^{7} \mathrm{Li}+{ }^{58} \mathrm{Ni}$, are compared with fresco CDCC calculations. Solid lines refer to uncoupled calculation while the dashed lines represent coupling channel calculations to continuum.

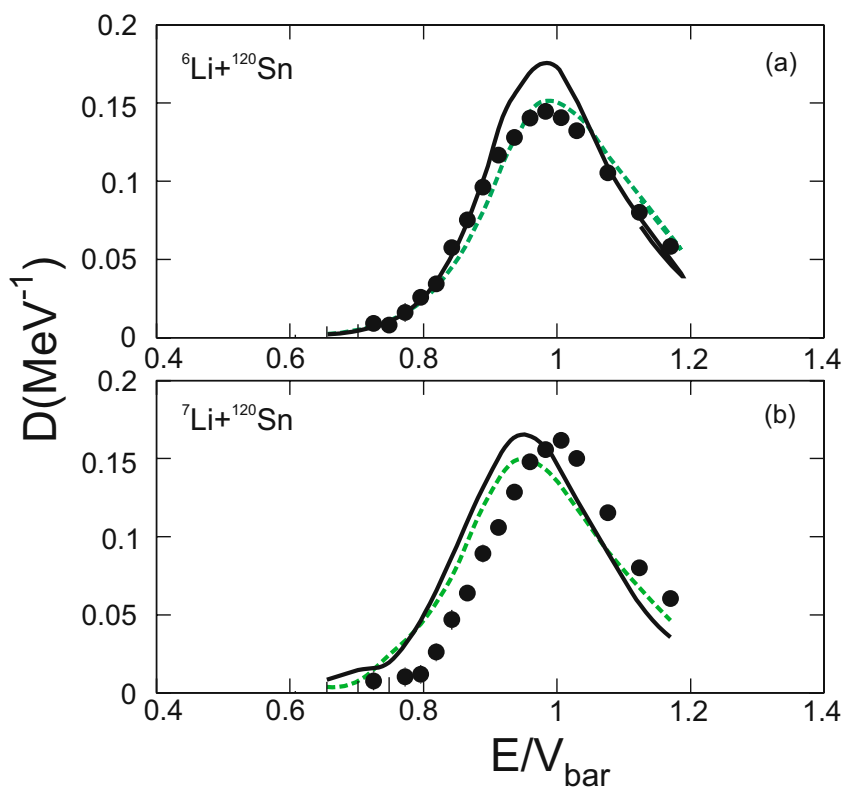

Fig. 13. (Color online) Experimental data of barrier distributions for (a) ${ }^{6} \mathrm{Li}+{ }^{120} \mathrm{Sn}$ and (b) ${ }^{7} \mathrm{Li}+{ }^{120} \mathrm{Sn}$, are compared with fresco CDCC calculations. Solid lines refer to uncoupled calculation while the dashed lines represent coupling channel calculations to continuum.

\section{In summary.}

We have determined barrier distributions via elastic backscattering for ${ }^{6,7} \mathrm{Li}$ on ${ }^{58} \mathrm{Ni},{ }^{116,120} \mathrm{Sn}$ and ${ }^{208} \mathrm{~Pb}$. Optical potentials based on the dispersion relation were probed at near- and sub-barrier energies indicating a loss of flux

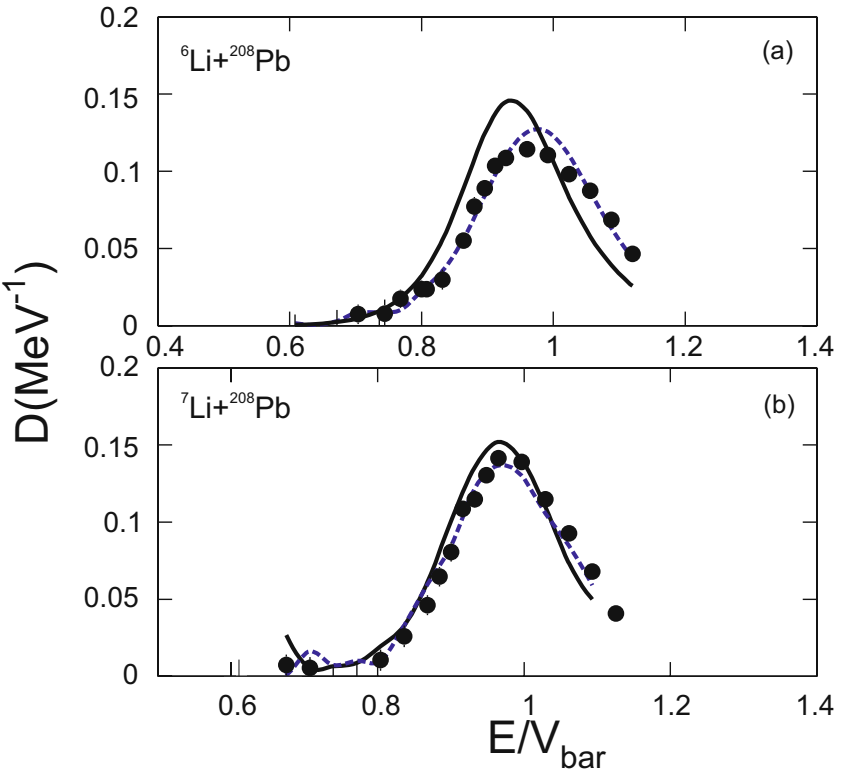

Fig. 14. (Color online) Experimental data of barrier distributions for $(\mathrm{a}){ }^{6} \mathrm{Li}+{ }^{208} \mathrm{~Pb}$ and (b) ${ }^{7} \mathrm{Li}+{ }^{208} \mathrm{~Pb}$, are compared with fresco CDCC calculations. Solid lines refer to uncoupled calculation while the dashed lines represent coupling channel calculations to continuum.

till very low energies for both weakly bound projectiles, although the energy dependence varies between them. Further on, the energy dependence between different targets but the same projectile is unique, possibly due to the involvement of different reaction mechansims, therefore different polarization potentials. The validity of the dispersion relation was questioned but not clearly concluded. The new systematic results indicate that the backscattering technique, by forming barrier distributions, is valuable tool for probing the potential at very low sub-barrier energies, acting complementary to conventional angular distribution and reaction cross-section measurements. Such results can also indicate the importance of coupling to the continuum without, however, giving clear insight into the reaction mechanism.

We would like to warmly acknowledge the personnel of the tandem at LNS-Catania for providing excellent beam conditions. We would also like to warmly acknowledge Massimo Loriggiola from LNL-Italy for providing the ${ }^{58} \mathrm{Ni},{ }^{120} \mathrm{Sn}$ and ${ }^{208} \mathrm{~Pb}$ targets. This research project is co-funded by the European Union - European Social Fund (ESF) National Sources, in the framework of the program "HRAKLEITOS II" of the "Operational Program Education and Life Long Learning" of the Hellenic Ministry of Education, Life Long Learning and religious affairs.

Open Access This is an open access article distributed under the terms of the Creative Commons Attribution License (http://creativecommons.org/licenses/by/3.0), which permits unrestricted use, distribution, and reproduction in any medium, provided the original work is properly cited. 


\section{References}

1. M. Dasgupta, D. Hinde, N. Rowley, A. Stefanini, Annu. Rev. Nucl. Part. Sci. 48, 401 (1998).

2. L.F. Canto, P.R.S. Gomes, R. Donangelo, M.S. Hussein, Phys. Rep. 424, 1 (2006).

3. N. Keeley, R. Raabe, N. Alamanos, J.L. Sida, Prog. Part. Nucl. Phys. 59, 579 (2007).

4. N. Keeley, N. Alamanos, K.W. Kemper, K. Rusek, Prog. Part. Nucl. Phys. 63, 396 (2009).

5. N. Rowley, G.R. Satchler, P.H. Stelson, Phys. Lett. B 254, 25 (1991)

6. J. Lubian et al., Nucl Phys. A 791, 24 (2007).

7. N. Rowley et al., Phys. Lett. B 373, 23 (1996).

8. K. Zerva et al., Phys. Rev. C 80, 017601 (2009).

9. K. Zerva et al., Phys. Rev. C 82, 044607 (2010).

10. I.I. Gontchar, D.J. Hinde, M. Dasgupta, J.O. Newton, Nucl. Phys. A 722, 479c (2003).

11. K. Hagino, T. Takehi, A.B. Balantekin, N. Takigawa, Phys. Rev. C 71, 044612 (2005).

12. K. Washiyama, K. Hagino, M. Dasgupta, Phys. Rev. C 73, 034607 (2006).

13. L.R. Gasques et al., Phys. Rev. C 76, 024612 (2007).

14. O.A. Capurro et al., Phys. Rev. C 75, 047601 (2007).

15. M. Evers et al., Phys. Rev. C 78, 034614 (2008).

16. J.F.P. Huiza et al., Phys. Rev. C 82, 054603 (2010).

17. E. Crema et al., Phys. Rev. C 84, 024601 (2011).

18. A. Pakou et al., Phys. Lett. B 556, 21 (2003).

19. A. Pakou et al., Phys. Rev. C 69, 054602 (2004).

20. C.J. Lin et al., Nucl. Phys. A 787, 281c (2007).
21. D.S. Monteiro et al., Phys. Rev. C 79, 014601 (2009).

22. D.R. Otomar et al., Phys. Rev. C 80, 034614 (2009).

23. S. Mukherjee et al., Phys. Rev. C 80, 014607 (2009).

24. D.T. Khoa et al., Phys. Lett. B 342, 6 (1995).

25. H.D. Vries, C.W. Jager, C. De Vries, At. Data Nucl. Data Tables 14, 479 (1974).

26. K.H. Bray et al., Nucl. Phys. 189, 35 (1972).

27. L. Trache et al., Phys. Rev. C 61, 024612 (2000) and private communication.

28. J. Raynal, Phys. Rev. C 23, 2571 (1981).

29. I.J. Thompson, Comput. Phys. Rep. 7, 167 (1988).

30. G.R. Satchler, Phys. Rep. 199, 147 (1991).

31. K.O. Pfeiffer, E. Speth, K. Berthge, Nucl. Phys. A 206, 545 (1973).

32. E.F. Aguilera et al., Phys. Rev. C 79, 021601(R) (2009).

33. N. Keeley et al., Nucl. Phys. A 571, 326 (1994).

34. N.N. Deshmukh et al., Phys. Rev. C 83, 024607 (2011).

35. M. Biswas et al., Nucl. Phys. A 802, 67 (2008).

36. A. Gómez Camacho et al., Nucl. Phys. A 833, 156 (2010).

37. M.A. Nagarajan, C.C. Mahaux, G.R. Satchler, Phys. Rev. Lett. 54, 1136 (1985).

38. P.R. Christensen, A. Winther, Phys. Lett. B 65, 19 (1976).

39. C.M. Perey, F.G. Perey, At. Data Nucl. Data Tables 17, 1 (1976).

40. R.P. Ward, P.R. Hayes, At. Data Nucl. Data Tables 49, 315 (1991).

41. N. Keeley, K. Rusek, Phys. Lett. B 427, 1 (1998).

42. C.M. Perey, F.G. Perey, Phys. Rev. 132, 755 (1963).

43. E.R. Flynn, D.D. Armstrong, J.G. Berry, A.G. Blair, Phys. Rev. 182, 1113 (1969). 